\title{
SIGNIFICANCE OF BODY MASS INDEX CHANGE DURING CONCOMITANT CHEMO RADIATION IN LOCALLY ADVANCED CERVICAL CANCER
}

O. Kaabia1, G. Nourallah¹, L. Aymen¹, Z. Rym¹, B. Mohamed¹, K. Hédi¹, B. Slim², B. Noureddine ${ }^{3}$. 1 Université de Sousse- Faculté de Médecine de Sousse- Hopital Farhat Hached- LR12ES03- 4000Sousse- Tunisia

2 Department of medical Oncology, Hopital Farhat Hached- 4000- Sousse- Tunisia,

3 Department of Radiotherapy - Farhat Hached Teaching Hospital, Sousse, Tunisia.

\section{Introduction :}

The body mass index (BMI) and simple counts of weight are easy and available tools in the clinic and in research in oncology. Recent studies have shown that BMI can influence the outcome of cervical cancer adjuvant treatment.

This study aims to evaluate the influence of BMI changes during concomitant chemotherapy and radiotherapy followed by an extrafacial hysterectomyon oncologic and surgical outcomes in women with locally advanced cervical cancer.

\section{Methods :}

This is a monocentric prospective study including 106 patients with locally advanced cervical carcinoma (FIGO Stage IB2 and more) that were allocated concomitant chemotherapy and radiotherapy (CCR) with an inclusion period from 2000 to 2009 and a follow up until 2018 in Farhat Hached Teaching Hospital, Sousse Tunisia. We calculated the BMI of the patients on the first day of each therapy session and on the day of the final MRI and on the day of the surgery.

\section{Results :}

The mean $\mathrm{BMI}$ at baseline was $35.1 \pm 5.6$ versus $33.9 \pm 4.5 \mathrm{~kg} / \mathrm{m}^{2}$ on the day of the final MRI with no statistically significant difference $(P=0.046)$.

The figure 1 shows the weight change during the CCR.
Eleven patients (10.4\%) had $10 \%$ or more weight loss and 20 patients $(18.9 \%)$ had $10 \%$ or more weight gain during the CCR sequence.

A pre-treatment BMI of $\geq 25 \mathrm{~kg} / \mathrm{m}^{2}$ was significantly associated with a higher complete response rate to the CCR $(P=0.03)$ and a lower 10 year recurrence rate $(\mathrm{P}=0.043)$.

A post-treatment BMI of $\geq 25 \mathrm{~kg} / \mathrm{m}^{2}$ was a significant factor for low recurrence rate $(P=0.049)$.

However, weight change during CCR was not significantly associated with complete response or 10 years recurrence rate. Pre and post-treatment BMls changes were not associated with lower surgical complications rates.

\section{Discussion :}

Many recent studies suggest that a high BMI is clinically associated with a higher cervical cancer risk.

Our study showed that a higher BMI before the CCR sequence in locally advanced cervical cancer candidate for a surgery is associated with a higher complete response rate to the CCR and a lower 10 years recurrence rate.

\section{Conclusion:}

The BMI variation in patients undergoing CCR for locally advanced cervical cancer may alter the response and the prognosis, with no impact on surgical complications

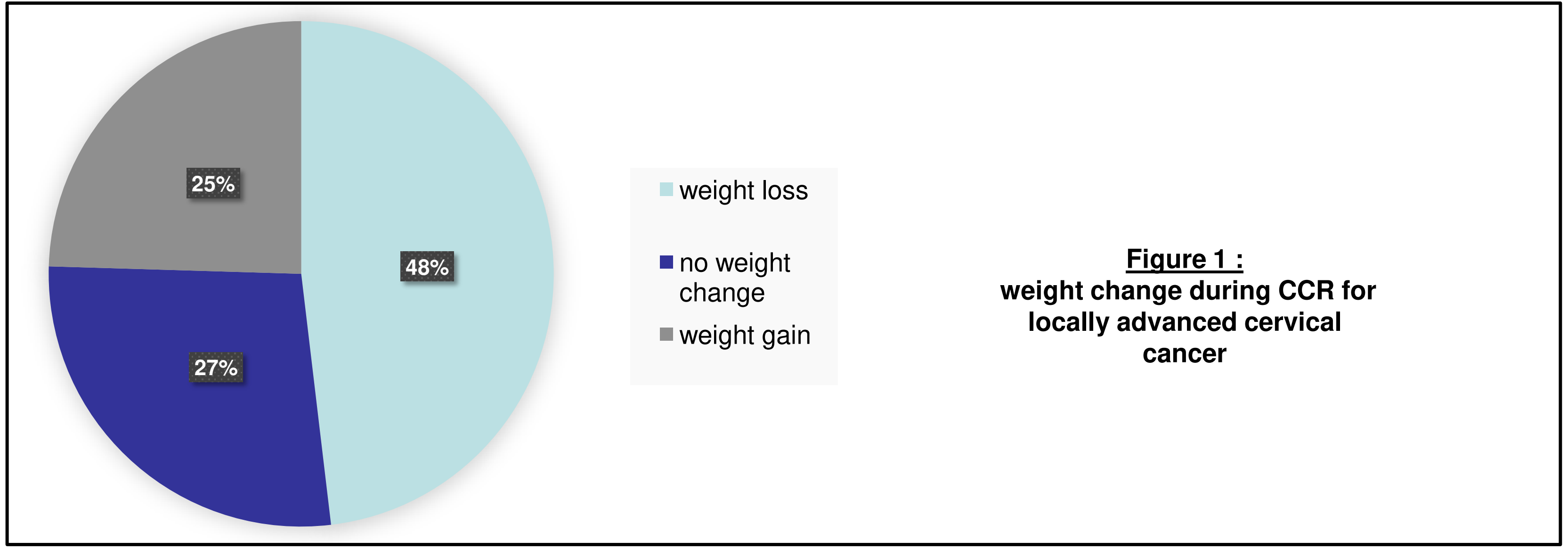

1. The association between BMI and cervical cancer risk: a meta-analysis. - PubMed - NCBI [Internet]. 